\title{
Bestimmung abnorm hoher Salzgehalte in seewasserähnlichen Salzlösungen ${ }^{1}$
}

\author{
Max GILlbRichT
}

Biologische Anstalt Helgoland, Zentrale, Hamburg 50

\begin{abstract}
Determination of abnormally high salinities in salt solutions similar to seawater. In the open sea the proportion between the different ions is rather constant with the exception of some elements influenced in their amount and distribution by biological activities $(\mathrm{P}, \mathrm{N}$, etc.). This relative constancy is modified if sea-water is diluted near the shores by river water, or concentrated by evaporation, especially in lower latitudes (e. g., Mediterranean, Red Sea). Abnormalities are also found in small isolated bodies of water near the shore. In all these instances the quantitative relation between the different ions is no longer constant; in most cases, calcium shows the first and most pronounced aberrations. It is desirable, therefore, to determine the individual components in such waters, not only its total salinity. In some cases, however, it may be of interest to measure high salinities as such, especially in biological studies. This paper describes a simple method for the determination of salinities between 50 and $275 \%$ via density measurements. The volume of the water sample is then determined by means of a $50 \mathrm{~cm}^{3}$-Knudsen pipette, its temperature measured, and the sample transferred into a clean, dry glass stoppered bottle. Thereafter the pipetted water is weighed and the $\alpha$ value (similar to the well known $\sigma_{t}$ ) calculated:
\end{abstract}

$$
\alpha=\left(\frac{\text { weight in air of the water }}{\text { volume of the pipette at } 20^{\circ} \mathrm{C}}-1\right) \times 1000
$$

In this formula, $\alpha$ and $\mathfrak{t}^{0} \mathrm{C}$ give the resulting salinity. This rather complicated procedure is simplified by the use of nomograms (at the end of this paper). The accuracy is not much better than $\pm 1 \%$. In situations in which it may be assumed that the $\mathrm{Cl} / \mathrm{S}$ ratio is practically identical with that in normal sea-water, $\mathrm{S} \%$ can be calculated from the value $\mathrm{g} \mathrm{Cl} / 1$; in this case the accuracy is $\pm 0.1 \%$.

\section{EINLEITUNG}

Der Salzgehalt der freien See schwankt nur sehr wenig um $35 \%$, und das Verhältnis der verschiedenen Ionen ist mit Ausnahme weniger biologisch beeinflußter Elemente ( $P, N$ etc.) recht konstant. Viele Jahrzehnte lang diente daher die Bestimmung des Chlorids (einschließlich des Bromids und Jodids) zur Ermittlung des Gesamtsalzgehalts, wobei man stillschweigend die schon frühzeitig erkannte und durch neuere Untersuchungen bestätigte Tatsache in Kauf nahm, daß auch hier mit durchaus meß-

${ }^{1}$ Herrn Professor Dr. FRIEDRICH KRüger zum 65. Geburtstag am 18. August 1967 in Verehrung gewidmet. 
baren Ionenverschiebungen ( $\mathrm{Ca}^{*}$ ) $\mathrm{zu}$ rechnen ist (Cox 1963), denen aber für diese yorzugsweise für biologische $Z_{w}$ ecke bestimmte Arbeit keinerlei Bedeutung zukommt.

Anders sieht es schon aus, wenn wir es mit ausgesüßtem Küstenwasser zu tun haben. Hier sagt der Gesamtsalzgehalt nur noch bedingt etwas über den Anteil der einzelnen Ionen aus. Der Einfluß der Salze auf Lebewesen ist aber sehr komplex, und neben dem durch die Gesamtmenge gegebenen osmotischen Wert spielen auch Ionenrelationen und die Mengen an bestimmten Salzen eine wichtige Rolle (SCHLIEPER \& Kow ALsKi 1956). Wir müssen im Brackwasser vor allem mit relativ zu hohen Werten für Kalzium und Alkalinität rechnen. Diese Abweichungen können nennenswerte biologische Wirkungen haben und müssen daher unter Umständen besonders bestimmt werden.

Weitaus schwieriger werden die Verhältnisse aber, wenn erheblich höhere Salzgehalte auftreten. Dies läßt sich wegen der großen Verdunstung in den niederen Breiten in geringem Ausmaße an der Oberfläche der Ozeane und schon stärker ausgeprägt in den warmen Rand- und Nebenmeeren beobachten. Diese Eindunstung führt in abgeschlossenen Becken zum Ausfallen der schwerer löslichen Salze, vor allem von $\mathrm{CaCO}_{3}$. So werden zum Beispiel abgestorbene Riffkorallen rasch mit einem dicken Kalkpanzer überzogen, und die Lücken $z$ wischen den alten Stöcken füllen sich ebenfalls mit Kalk. In Strandtümpeln etc. geht die Eindunstung noch erheblich weiter. Wenn es hierbei auch zu starken Übersättigungen kommen kann - die Salze fallen dann erst beim Schöpfen aus (persönliche Mitteilung von Herrn Dr. I. Kristensen) - so ist in solchen Bereichen der Begriff des "Seewassers" doch immer recht fragwürdig. Daher kann auch der Salzgehaltswert nur noch mit Einschränkungen angegeben werden. Mit steigender Konzentration wird es wünschenswert, die vorkommenden Ionen gesondert zu bestimmen. Als Beispiel für dieses Problem sei hier die Zusammensetzung des wegen seiner fragwürdigen Herkunft vielleicht besonders abweichenden warmen Tiefenwassers des Roten Meeres gebracht:

Tabelle 1

Salzgehalt des warmen Tiefenwassers des Roten Meeres

\begin{tabular}{|lcll|}
\hline Cl\%o & $\begin{array}{c}\text { S\%o } \\
\text { aus Cl\%o berechnet }\end{array}$ & \multicolumn{1}{c}{$\begin{array}{c}\text { S\%o } \\
\text { gemessen }\end{array}$} & \multicolumn{1}{c|}{ Autoren } \\
\hline 181 & 327 & 326,5 & Krause \& ZIEGENBEIN (1966) \\
154,28 & 278,51 & 254,94 & BREWER, RLLEY \& CULKIN (1965) \\
\hline
\end{tabular}

Die zweite Probe ist genauer analysiert worden, wobei sich vor allem ein Defizit an $\mathrm{Mg}^{\prime}$ und $\mathrm{SO}_{4}$ " herausstellte. Der Unterschied der beiden Ergebnisse in bezug auf die Cl/S-Relation kann methodisch bedingt sein, da im ersten Falle S\% $\% 00$ mit einem induktiven Salinometer und im zweiten gravimetrisch bestimmt wurde. Beide Verfahren geben aber nach den Messungen von Morris \& RiLeY (1964) bei Ionenverschiebung durchaus verschiedene Resultate, wobei die Werte der Chloridtitration sich zumeist mehr mit denen der Leitfähigkeitsmessung als mit denen der gravimetrischen Methode decken, wie ein Beispiel aus der Ostsee deutlich ergibt (MORRIs \& RILEY 1964): 
$\mathrm{S} \%$ aus $\mathrm{Cl} \%$

20,145
$\mathrm{S} \%$ aus Leitfähigkeit 20,136
S*/00 gravimetrisch 20,197

Den Einfluß von Beimischung und Verdunstung auf die Beziehung zwischen S\% 00 aus $\mathrm{Cl} \%$ und $\mathrm{S} \%$ gravimetrisch zeigt die Untersuchung von Morcos \& RiLex (1966) aus dem Gebiet des Suez-Kanals mit dem Süßwassereinfluß des Nils und den Salzlagern in den Bitterseen (Tab. 2).

Tabelle 2

Salzgehalts-Mittelwerte aus dem Suez-Gebiet

\begin{tabular}{|lccc|}
\hline Lokalizät & $\begin{array}{c}\text { Anzahl } \\
\text { Messungen }\end{array}$ & S \%o aus C1 \%o & $\begin{array}{c}\text { S \%o } \\
\text { gravimetrisch }\end{array}$ \\
\hline Vom Nilwasser beeinflußt & 4 & 34,471 & 34,499 \\
Bucht von Suez & 6 & 42,568 & 42,476 \\
Bitterseen & 7 & 45,446 & 45,335 \\
\hline
\end{tabular}

Jedoch ist es oft nützlich, trotz aller Bedenken den Gesamtsalzgehalt angeben zu können. Eine solche Bestimmung ist grundsätzlich nicht problematisch. Nach hinreichender Verdünnung läßt sich der Salzgehalt mit den üblichen Verfahren (Leitfähigkeit, Induktivität, Dichte, Chloridtitration) ermitteln.

Nun ist aber der Biologe an Arbeiten in einem durch hohe Salzgehalte extremen Lebensraum aus mancherlei Gründen interessiert. Infolgedessen fallen hier bei Freilandbeobachtungen und in Experimenten relativ viele derartige Messungen an, und besonders für diese $Z_{\text {wecke }}$ ist ein einfaches Bestimmungsverfahren wünschenswert.

\section{METHODIK UND ERGEBNISSE}

Im Normalbereich von $O$ bis $40 \%$ bietet sich für die Salzgehaltsbestimmung bei nicht zu hohen Ansprüchen der in der Meereskunde übliche Aräometersatz an (KNUDSEN 1901, GILLBRICHT 1959). Es ist zunächst naheliegend, diese Methode auch bei extrem hohen Werten anzuwenden. Dagegen sprechen zwei Gründe: (1) Entsprechende Aräometer sind nicht handelsüblich, und ihre Beschaffung dürfte daher auf Schwierigkeiten stoßen. (2) Um mit einer vertretbaren $\mathrm{Zahl}$ von Aräometern auszukommen, muß der Meßbereich jeweils sehr groß sein. Das hat vor allem wegen der wechselnden Oberflächenspannung der Proben eine sehr geringe Genauigkeit zur Folge (Goedeckf \& GILlbricht 1967).

Da die Mohrsche Waage heute kaum noch in einem biologischen Laboratorium anzutreffen und sicherlich noch weniger ein mit ihrer Handhabung wirklich vertrauter Laborant vorhanden ist, dürfte auch dieser Weg aus praktischen Erwägungen entfallen. Eine einfache Dichtemessung scheint aber nichtsdestoweniger für unsere Zwecke am günstigsten zu sein. Das setzt jedoch voraus, daß die Dichte des "Seewassers" bei abnorm hohen Salzgehalten zunächst überhaupt erst einmal bestimmt wird.

Da die Hydrographischen Tabellen (KNUDSEN 1901) nur wenig über 40\% S gehen und später bis $47 \%$ (Morcos 1960) und hinsichtlich der Dichte sogar bis 54,5\%00 
extrapoliert wurden (Density table ... 1945), erschien es zweckmäßig, mit der Dichtemessung bei $50 \% \mathrm{zu}$ beginnen.

Die Untersuchung wurde mit künstlichem Seewasser durchgeführt, indem im richtigen Mengenverhältnis folgende Substanzen in Wasser gelöst wurden $\left(\mathrm{MgCl}_{2}\right.$ wurde als nicht wägbar in eingestellter Lösung zugesetzt):

$\begin{array}{ll}\mathrm{NaCl} & \mathrm{NaHCO}_{3} \\ \mathrm{MgSO}_{4} \cdot 7 \mathrm{H}_{2} \mathrm{O} & \mathrm{KBr} \\ \mathrm{CaCl}_{2} \cdot 2 \mathrm{H}_{2} \mathrm{O} & \mathrm{H}_{3} \mathrm{BO}_{3} \\ \mathrm{KCl} & \mathrm{MgCl}_{2}\end{array}$

Der Salzgehalt in den einzelnen Proben wurde durch Verdünnen und Messen mit einem induktiven Salinometer bestimmt. Ab 150\% bildeten sich nennenswerte Niederschläge, die getrocknet gewogen wurden. Ihre Menge ist in $\% / 0$ der wasserfreien Salze angegeben (Tab. 3).

Tabelle 3

Prozentuale Mengen an Niederschlägen bei der Herstellung von hohen Salzgehalten

\begin{tabular}{|cc|}
\hline $\mathrm{S} \% 0$ & Fällung in \% der Salzmenge \\
\hline 50,07 & - \\
100,69 & - \\
150,93 & 1,6 \\
202,73 & 4,6 \\
250,50 & 6,2 \\
274,19 & 18,2 \\
\hline
\end{tabular}

Diese Niederschläge beeinflussen naturgemäß durch die hierdurch unvermeidlichen Ionenverschiebungen die ganzen Messungen. Entsprechend wird auch der Zusammenhang zwischen Salzgehalt und Dichte unsicher, weshalb sich sehr präzise Untersuchungen erübrigen. Daher wurde auch nur mit dem Meßaufwand gearbeitet, mit dem später die Bestimmungen durchgeführt werden sollen. Die Proben wurden hierzu bei verschiedenen Temperaturen mit einer sehr gut geeichten Knudsen-Pipette von $\mathrm{ca} .50 \mathrm{~cm}^{3}$ Inhalt sorgfältig in saubere und trockene Glasstopfenflaschen pipettiert und dann gewogen (erforderliche Genauigkeit dieser Wägungen mindestens $10^{-2} \mathrm{~g}$ ). Aus dem Gewicht in Luf, dem Luftauftrieb und dem für die jeweilige Temperatur korrigierten Pipettenvolumen ergibt sich dann die Dichte. Hieraus errechnet sich das in der Meereskunde übliche $\sigma_{\mathrm{t}}$ nach folgender Formel:

$$
\sigma_{\mathrm{t}}=(\text { Dichte }-1) \times 1000
$$

Man schreibt also beispielsweise statt Dichte $=1,0297$ stets $\sigma_{\mathrm{t}}=29,7$.

Diese $\sigma_{t}$-Werte wurden für alle sechs Salzlösungen bei den Temperaturen $10^{\circ}, 15^{\circ}$, $20^{\circ}, 25^{\circ}$ und $30^{\circ} \mathrm{C}$ jeweils viermal bestimmt, wobei die exakte Temperatur für jede Probe gesondert gemessen wurde. Von diesen 120 Werten wurden 18 als mit den andern nicht hinreichend übereinstimmend verworfen. Nun ist das Pipettieren auch bei der Chloridtitration Minimumfaktor (Cox 1963), so daß dies Ergebnis nicht weiter verwundert. 
Aus den verbleibenden 102 Ergebnissen läßt sich eine Gleichung berechnen, die den Zusammenhang $z$ wischen $\sigma_{\mathrm{t}}, \mathrm{t}^{0}$ und $\mathrm{S}^{0} / 00$ hinreichend beschreibt:

$$
\begin{aligned}
& \sigma_{\mathrm{t}}=-4,88-0,028 \mathrm{t}-0,00030 \mathrm{t}^{2}-0,000056 \mathrm{t}^{3}+0,9352 \mathrm{~S}-0,000940 \mathrm{~S}^{2} \\
& +0,00000211 \mathrm{~S}^{3}-0,00317 \mathrm{tS}+0,0000056 \mathrm{t}^{2} \mathrm{~S}+0,00000624 \mathrm{tS}^{2}
\end{aligned}
$$

Diese aufwendige Formel ist notwendig, um über einen so großen Bereich befriedigende Übereinstimmung zwischen Messung und Rechnung zu erzielen, wobei sich aber immer

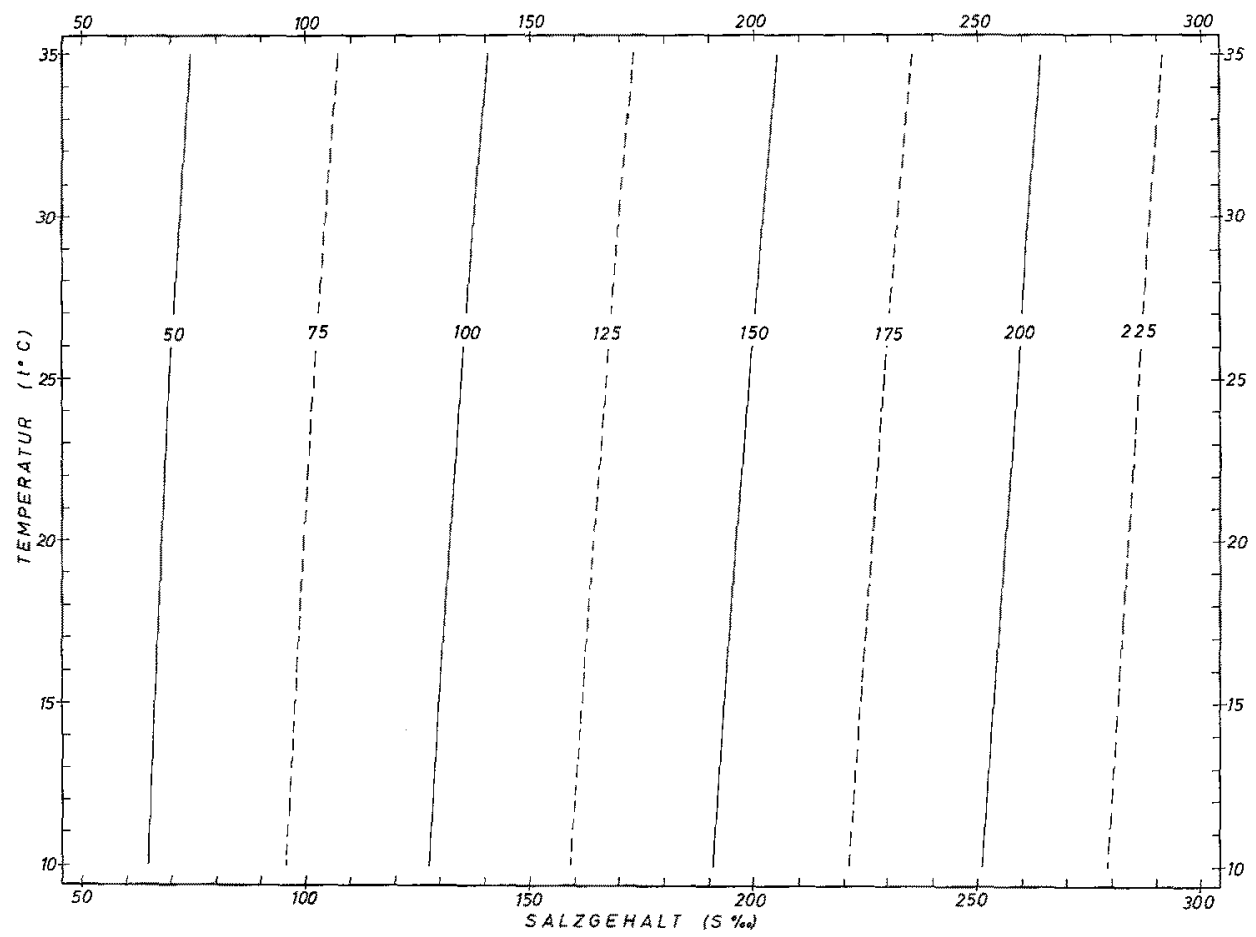

Abb. 1: $\sigma_{t}$ einer seewasserähnlichen Salzlösung als Funktion von Temperatur und Salzgehalt

noch gewisse systematische Abweichungen beobachten lassen. Bei 50,07\% liegt beispielsweise das berechnete $\sigma_{\mathrm{t}}$ im Durchschnitt um 0,10 Einheiten niedriger als das beobachtete, während wiederum die Hydrographischen Tabellen (KNUDSEN 1901) einen um 0,12 Einheiten zu hohen Wert liefern. Ob diese letztere Abweichung an einer nicht mehr zulässigen Extrapolation der alten Tabellen liegt, ob systematische Unterschiede vorhanden sind, oder ob unsere für weniger hohe Ansprüche bestimmte Untersuchung einfach keine bessere Übereinstimmung erwarten läßt, bleibt dahingestellt. Immerhin sind mit diesen Werten schon die Grenzen des Verfahrens abgesteckt. In Abbildung 1 ist der Zusammenhang $z$ wischen $\sigma_{t}, t^{0}$ und $S \%$ dargestellt.

Die Bestimmung von $\sigma_{\mathrm{t}}$ durch Auswiegen eines Pipetteninhalts erfordert eine erhebliche Rechenarbeit, die sich weitgehend vermeiden läßt, wenn nicht $\sigma_{t}$, sondern nur S\%oo gesucht wird. Dann ergibt sich der Salzgehalt direkt aus der Temperatur beim 
Pipettieren, dem Luftgewicht des Wassers und dem Pipetteninhalt bei $20^{\circ} \mathrm{C}$. Wenn wir eine Umrechnung wie von Dichte auf $\sigma_{t}$ vornehmen und diesen Wert als $\alpha$ bezeichnen, so erhalten wir:

$$
\alpha=\left(\frac{\text { Luftgewicht des Wassers }}{\text { Pipettenvolumen bei } 20^{\circ} \mathrm{C}}-1\right) \times 1000
$$

Für den untersuchten Meßbereich ergibt sich folgende Gleichung:

$$
\begin{aligned}
\alpha= & -4,78-0,250 t+0,01134 t^{2}-0,000231 t^{3}+0,9327 S-0,000941 S^{2} \\
& +0,00000212 S^{3}-0,00299 t S+0,0000031 t^{2} S+0,00000616 t^{2}
\end{aligned}
$$

Mit ihrer Hilfe lassen sich Fluchtentafeln aufstellen, welche die Auswertung derartiger Bestimmungen erheblich vereinfachen. Diese sieben Nomogramme umfassen einen Bereich von $\mathrm{O}$ bis $360 \%$, wobei die erste Tafel $(\mathrm{O}$ bis $60 \%$ ) aus den Angaben in den Hydrographischen Tabellen (KNUDSEN 1901) berechnet wurde. Sie dient nur zur Ergänzung, falls versehentlich einmal ein relativ salzarmes Wasser nach dieser Methode untersucht wurde. Bewußt im niederen Bereich mit diesem Verfahren zu arbeiten, kann wegen der geringen Genauigkeit nicht empfohlen werden. Die Werte oberhalb 275\% sind extrapoliert worden, um notfalls auch hier noch gewisse Aussagen machen zu können. Die Temperaturangabe wurde bis $35^{\circ} \mathrm{C}$ erweitert.

Die Diagramme sind auf zwei am Ende dieser Arbeit eingefügten Falttafeln zusammengestellt, wobei die zusammengehörigen Skalen jeweils gleiche Farbe haben. Die Benutzung ist einfach: Die abgelesenen Werte für $\alpha$ und $t^{0}$ werden auf den Skalen gleicher Farbe aufgesucht und mit einem Lineal oder Zwirnsfaden miteinander ver-

Tabelle 4

Umrechnung von $\mathrm{g} \mathrm{Cl} / \mathrm{l}$ mit dem Faktor (F) zur Ermittlung des Salzgehaltes in $0 \%$ $\mathrm{S} \%=\mathrm{F} \cdot \mathrm{g} \mathrm{Cl} / \mathrm{I}$

\begin{tabular}{|cc|cc|cc|}
\hline $\mathrm{g} \mathrm{Cl} / 1$ & $\mathrm{~F}$ & $\mathrm{~g} \mathrm{Cl} / 1$ & $\mathrm{~F}$ & $\mathrm{~g} \mathrm{Cl} / 1$ & $\mathrm{~F}$ \\
\hline 20 & 1,7631 & 100 & 1,6045 & 180 & 1,4855 \\
25 & 1,7508 & 105 & 1,5964 & 185 & 1,4786 \\
30 & 1,7389 & 110 & 1,5884 & 190 & 1,4718 \\
35 & 1,7274 & 115 & 1,5805 & 195 & 1,4650 \\
40 & 1,7163 & 120 & 1,5727 & 200 & 1,4583 \\
45 & 1,7056 & 125 & 1,5650 & 205 & 1,4517 \\
50 & 1,6952 & 130 & 1,5574 & 210 & 1,4450 \\
55 & 1,6851 & 135 & 1,5499 & 215 & 1,4384 \\
60 & 1,6753 & 140 & 1,5425 & 220 & 1,4318 \\
65 & 1,6657 & 145 & 1,5352 & 225 & 1,4253 \\
70 & 1,6564 & 150 & 1,5279 & 230 & 1,4187 \\
75 & 1,6473 & 155 & 1,5207 & 235 & 1,4122 \\
80 & 1,6384 & 160 & 1,5135 & 240 & 1,4058 \\
85 & 1,6297 & 165 & 1,5064 & 245 & 1,3995 \\
90 & 1,6212 & 170 & 1,4994 & 250 & 1,3931 \\
95 & 1,6128 & 175 & 1,4924 & & \\
\hline
\end{tabular}

Beispiel: $\quad \mathrm{g} \mathrm{Cl} / 1=43,25 \quad \begin{aligned} \mathrm{F} & =1,7093 \\ \mathrm{~S}^{0} / 00 & =73,9\end{aligned}$ 


\section{Tafel 1 \\ M. GILLBRICHT :}

Fluchtentafel zur Bestimmung des Satzgehaltes durch Auswiegen einer Pipettenfüllung

(Beilage zu: „Helgoländer wissenschaftliche Meeresuntersuchungen”, Band 16, 1967)

$S=S$ alzgehalt, $\mathrm{t}^{0}=$ Wassertemperatur beim Pipettieren

$$
\alpha=\left(\frac{\text { Gewicht der Pipettenfülllung in Lutt }}{\text { Pipettenvolumen bei } 20^{\circ} \mathrm{C}}-1\right) \times 1000
$$

Zusammengehörige Maßsstäbe haben die gleiche Farbe

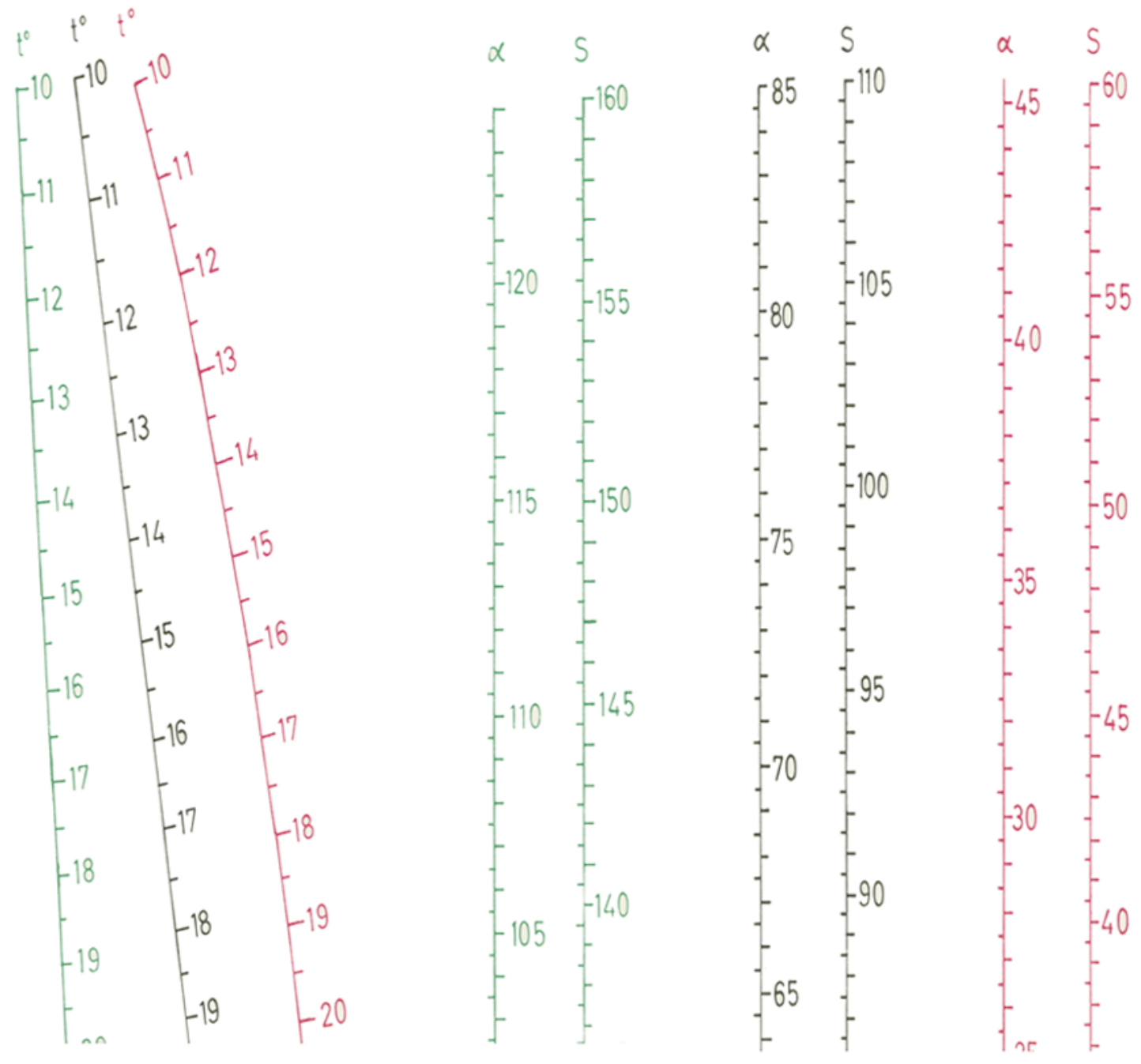



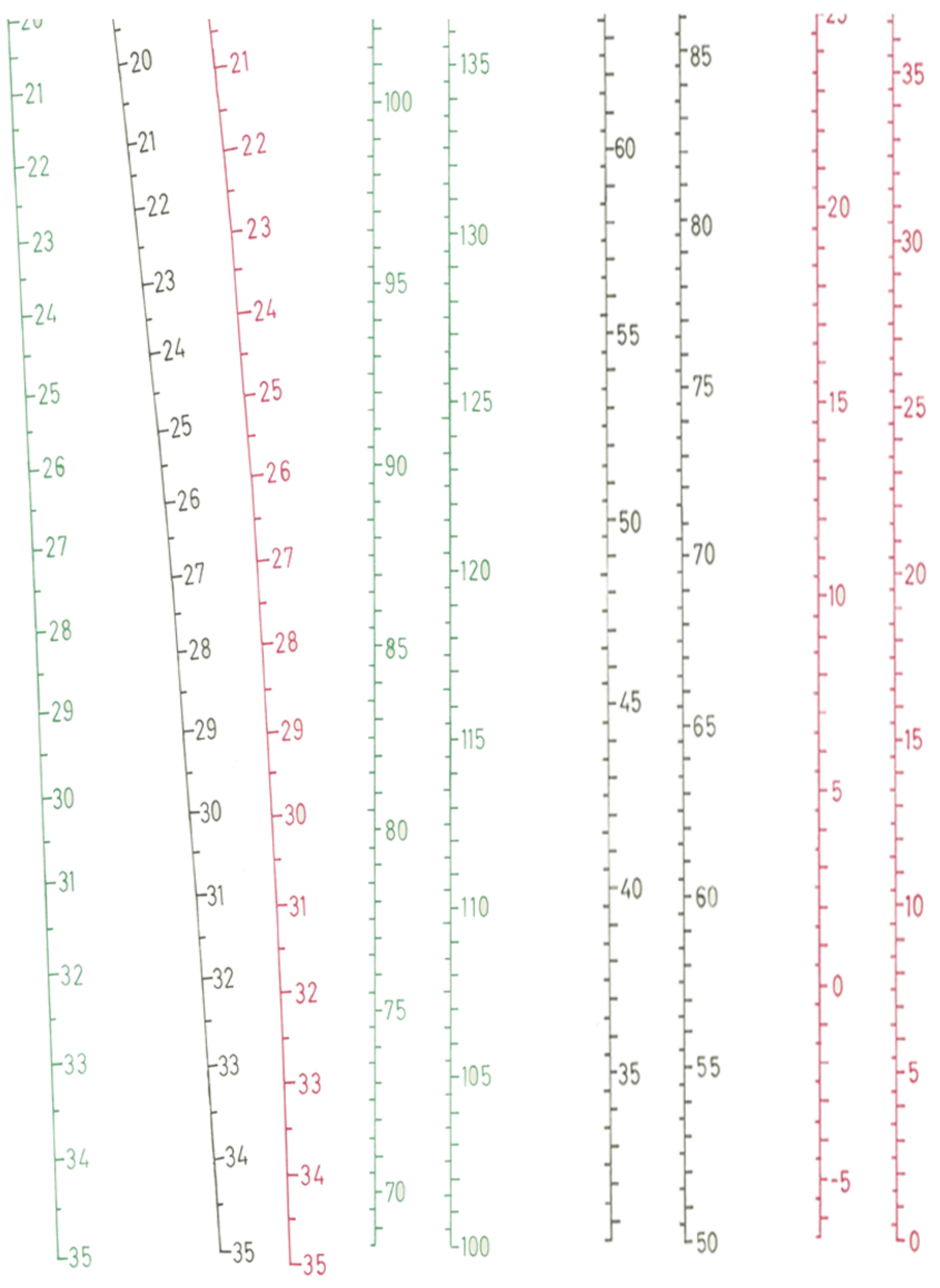
Tatel 2

\section{M.GILLRICHT:}

Fluchtentafele zur Bestimmung des Satzzehaltes durch Auswiegen einer Pipettenfiullung

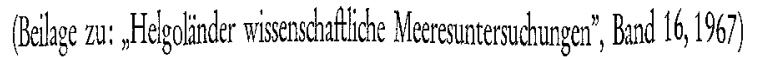

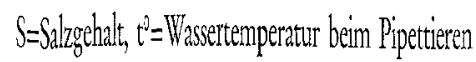

$\alpha=\left(\frac{\text { Cerridac cer Pipetentifillung in Lut }}{\text { Pipettentolumen be } 20^{\circ} \mathrm{C}}-1\right) \times 1000$

Zurammengehörge Malsstabe laben die gleche Farbe

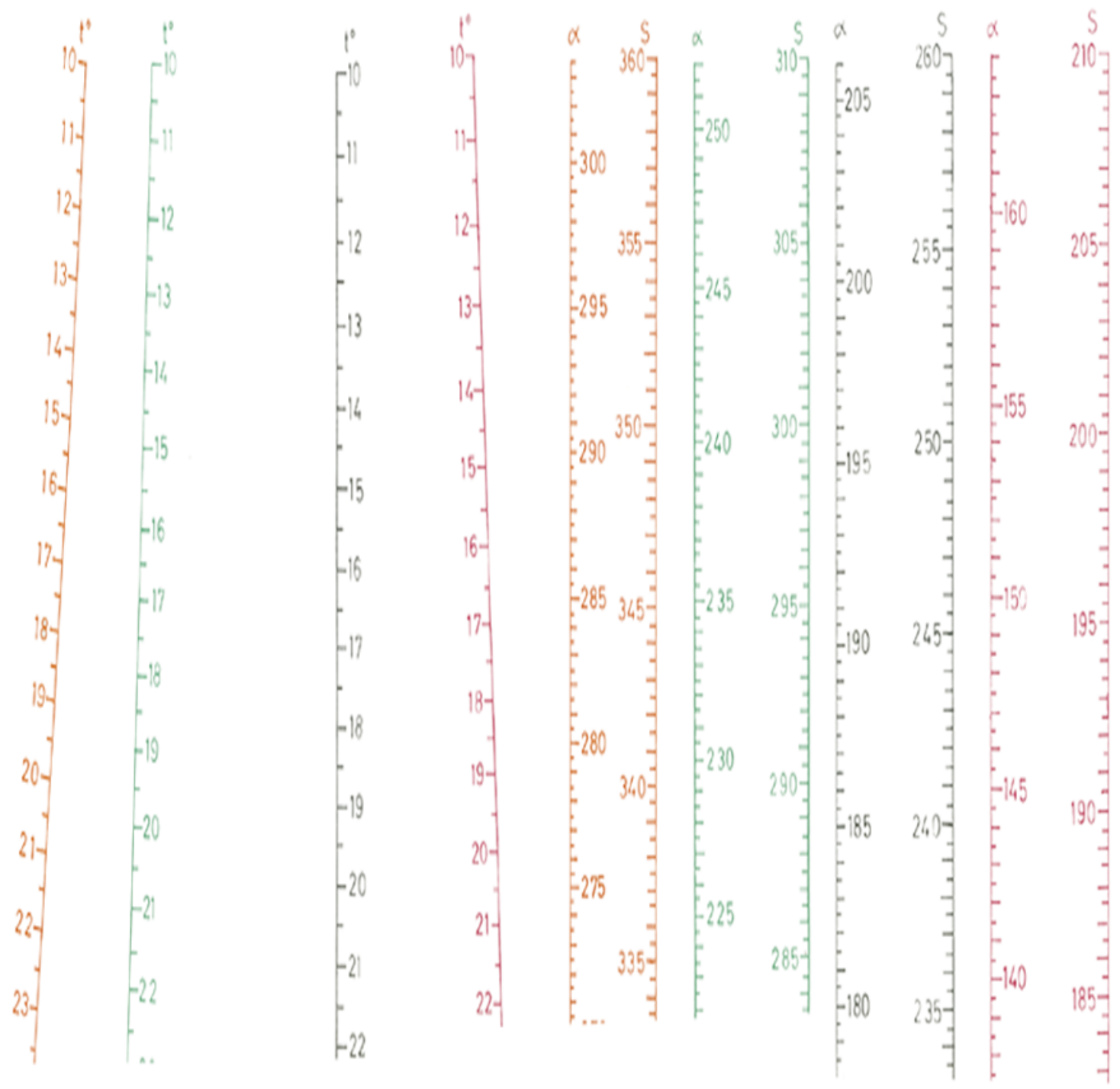




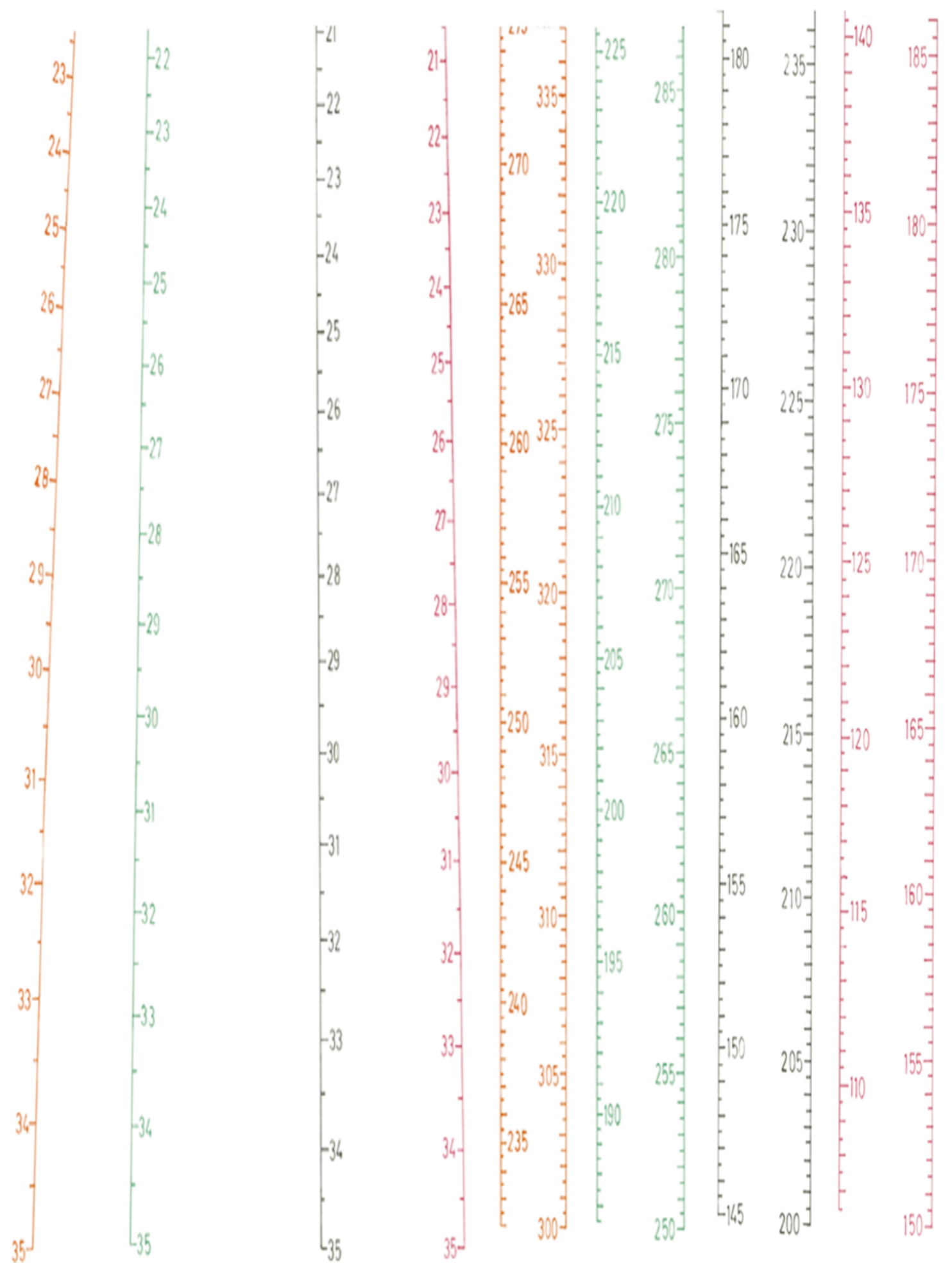


bunden. Die Verlängerung der Geraden gibt auf der dritten Skala gleicher Farbe den zugehörigen $\mathrm{S}^{0} / 00$-Wert an.

$$
\text { Beispiel: } \quad \begin{aligned}
\alpha & =62,0 \\
t^{0} & =22,2 \\
S^{0} / 00 & =85
\end{aligned}
$$

Die Genauigkeit der Methode ergibt sich auf Grund der eigenen 120 Messungen (also bei Einbeziehung der für die Rechnung nicht benutzten abweichenden Werte) bei einem Pipettenvolumen von $50 \mathrm{~cm}^{3} \mathrm{zu} \pm 0,6 \%$; folglich sollten die Ergebnisse nur auf $\pm 1 \%$ gebracht werden, da zwischen den Untersuchungspunkten noch mit gewissen systematischen Abweichungen zu rechnen ist. Vor allem bei den höheren Werten ist aber eine genauere Angabe mit Rücksicht auf die Fragwürdigkeit des vom normalen Seewasser stammenden Begriffs "Salzgehalt" auch gar nicht mehr sinnvoll.

Kann jedoch angenommen werden, daß die Cl/S-Relation noch dem Normalwert entspricht, dann erlauben die vorliegenden Dichtebestimmungen die Umrechnung von $\mathrm{g} \mathrm{Cl} / 1$ auf $\mathrm{S} \%$ mit einer Genauigkeit von $\pm 0,1 \% \mathrm{~S}$. Um die Tabelle klein zu halten, ist in größeren Abständen der Faktor $(F)$ angegeben, mit dem $\mathrm{g} \mathrm{Cl} / 1$ multipliziert werden muß, um zu S\% $/ 00$ zu kommen (Tab. 4).

\section{ZUSAMMENFASSUNG}

1. Die im normalen Seewasser recht konstante Ionenrelation kann bei niederen, vor allem aber bei extrem hohen Salzgehalten erheblich gestört sein. Daher ist in diesen Bereichen die Bestimmung der einzelnen Komponenten wünschenswert. Die Bezeichnung $\mathrm{S} \% 0$ ist bei sehr großen Salzmengen nur bedingt vertretbar, und übertrieben genaue Angaben sind daher wenig sinnvoll. Deshalb ist für viele Belange ein einfaches Meßverfahren hinreichend.

2. Es wird eine Methode beschrieben, mit deren Hilfe hohe Salzgehalte durch Auswiegen des Inhalts einer Knudsen-Pipette bestimmt werden können. Die Auswertung erfolgt mit Hilfe von Nomogrammen. Die Genauigkeit der Methode dürfte bei $\pm 1 \%$ liegen.

3. Die durchgeführten Dichtemessungen erlauben, bei Gültigkeit der $\mathrm{Cl} / \mathrm{S}$-Relation $\mathrm{g} \mathrm{Cl} / 1$ auf $\mathrm{S} \%$ mit einer Genauigkeit von $\pm 0,1 \%$ umzurechnen.

Herrn K. TREuTtNer (Meeresstation der BAH) danke ich für die Durchführung der Messungen und eines Teils der aufwendigen Rechnungen.

\section{ZITIERTE LITERATUR}

Brewer, P. G., RrLey, J. P. \& Culkin, F., 1965. The chemical composition of the hot salty water from the bottom of the Red Sea. Deep Sea Res. 12, 497-503.

Cox, R. A., 1963. The salinity problem. Progr. Oceanogr. 1, 243-261.

DENSITY table for sea-waters of high salinity and temperature 1945. In: Oceanographical information sheet. British Admiralty, Hydrogr. Dpt., H. D. No 482. 
Gillbricht, M,, 1959. Fluchtentafeln zur Bestimmung des Salzgehalts mit Hilfe des Seewasseraräometers. Helgoländer wiss. Meerestunters, 6, 239-240.

GoEdecke, E. \& GrlLbricht, M., 1967. Über den Einfluß der Oberflächenspannung des Seewassers auf Aräometermessungen (im Drudk).

Knudsen, M. (Hrsg.), 1901. Hydrographische Tabellen. Bianco Luno, Kopenhagen, 63 pp.

Krause, G. \& Zregenbein, J., 1966. Struktur des heißen salzreichen Tiefenwassers im zentralen. Roten Meer. In: "Meteor" Forschungsergebnisse. Hrsg. von der Dt. ForschGem. Reihe A. Borntraeger, Berlin, 1, 53-58.

Morcos, S. A., 1960. Hydrographical tables for high salinities. Bull. Inst. oceanogr. Monaco $57(1180), 1-27$.

- \& Rilex, J. P., 1966. Chlorinity, salinity, density and conductivity of sea water from the Suez Canal region. Deep Sea Res. 13, 741-749.

Morris, A. W. \& RrieY, J. P., 1964. The direct gravimetric determination of the salinity of sea water. Deep Sea Res. 11, 899-904.

Schlieper, C. \& Kowalski, R., 1956. Quantitative Beobachtungen über physiologische Ionenwirkungen im Bradkwasser. Kieler Meeresforsch. 12, 154-165. 